\title{
ARTICLE
}

Cite this: DOI: $10.1039 /$ xoxxooooox

\section{Delayed voltammetric with respect to amperometric electrochemical detection of concentration changes in microchannels}

Received ooth January 2012, Accepted ooth January 2012

DOI: $10.1039 /$ xoxxooooox

www.rsc.org/

\author{
Raphaël Trouillon and Martin A. M. Gijs
}

The time response of an electrode incorporated into a fluidic channel to variations in analyte concentration of the outer-sphere redox probe ferrocenemethanol was investigated, both for amperometry (AMP) and cyclic voltammetry (CV). The experimental data show that the temporal resolution of $\mathrm{CV}$ is not as good as for AMP, as $\mathrm{CV}$ cannot properly detect fast concentration transients. The delayed response of $\mathrm{CV}$ was previously reported, for neurotransmitters, and mostly attributed to adsorption of the analyte to the electrode surface. By using an outer-sphere redox couple, we show that mass transport also significantly delays the response of CV. The experimental delay time in CV was understood from mass transfer limitations due to the relaxation of the diffusion layer during repeated potential scanning. Furthermore, a robust protocol for the analysis of fast concentration transients was established, using the impulse and modulation transfer functions of the system. This method was found to be more precise than the mere analysis of the undifferentiated traces in the time domain. As a proof of concept, the effect of increased viscosity was investigated, showing that AMP was more sensitive than $\mathrm{CV}$ to these variations. Overall, this analysis underlines further the enhanced temporal sensitivity of AMP over CV, at the expense of decreased chemical resolution, potentially having implications for in situ electrochemical detection of biologically relevant molecules.

\section{Introduction}

Recent years have witnessed a tremendous development of micro-devices applied to bio-analysis. ${ }^{1}$ The micrometric scale of these systems is ideally suited to the small sample size often encountered in life sciences, and is also compatible with single cell analysis. ${ }^{2,3}$ Their micro-fabricated nature also makes them highly amenable to miniaturization, mass production and automation, a welcome feature when reproducibility, massively parallel capabilities and wide dissemination are all expected to facilitate the application of new biotechnologies to diagnostics, individual healthcare or routine laboratory analysis. These devices are commonly fabricated by soft lithography, ${ }^{4-6}$ and usually combine an analytical method, for the final assessment of the reaction of interest, with a fluidic system, used to handle and prepare the sample.

Even though the main detection scheme in these chips is often based on optics, electrochemical methods offer several attractive characteristics for on-chip analysis., ${ }^{7,8}$ Indeed, electrochemical stations can now be significantly miniaturized, and several commercial hand-held and/or Universal Serial Bus (USB)-powered options are available. Additionally, electrodes can be manufactured by standard microfabrication methods and are obviously complementary to microfluidic systems. Electrochemical detection of biomolecules has been routinely used for the analysis of several biological processes, e.g. neurotransmission, ${ }^{9,10}$ and have shown good sensitivities and detection capabilities for bio-applications. ${ }^{11,12}$ Furthermore, recent developments in the fields of tissue-, cells- or organ-ana-chip ${ }^{13-16}$ could take advantage of the versatility and ease of use of electrochemical sensors. Indeed, the biological use, in situ, of electrochemical techniques is now well-known, and integrating these systems into these new devices is now an obvious step to achieve a reliable, cheap and user-friendly biochip with integrated detection. Again, the specificity of electrochemical detection would here be a welcome complement to the optical methods typically used in this field. These considerations have motivated the study of electrochemistry in fluidic channels. Indeed, electrochemistry is intrinsically based on the analysis of fluxes of analytes, and the specific diffusive and convective phenomena encountered in microchannels are expected to lead to new observations. The specificity of microfluidics may also induce new phenomena. For instance, electrochemistry has been found to resolve transient hydrodynamic flow profiles in microchannels. ${ }^{17}$ Similarly, the response of inlaid band microelectrodes can be significantly altered by the characteristics of the system. ${ }^{18-20}$ Previous reports focusing at the redox chemistry of dopamine have reported that fast-scan cyclic voltammetry (CV) shows a delayed response in comparison to amperometric (AMP) recordings. This delay was principally attributed to dopamine adsorption to the electrode surface, thus slowing the 
electrochemical response in the case of a fast potential ramp. ${ }^{21,22}$ However, the role of mass transport in this delay has not been fully investigated in this particular case. The specificity of CV over AMP, i.e. the rapid variation of the applied potential, allows for a better discrimination of the different analytes in solution, but could also be at the basis of a delayed response due to a continuously changing nonequilibrium depletion layer and associated relaxation effects near the electrode.

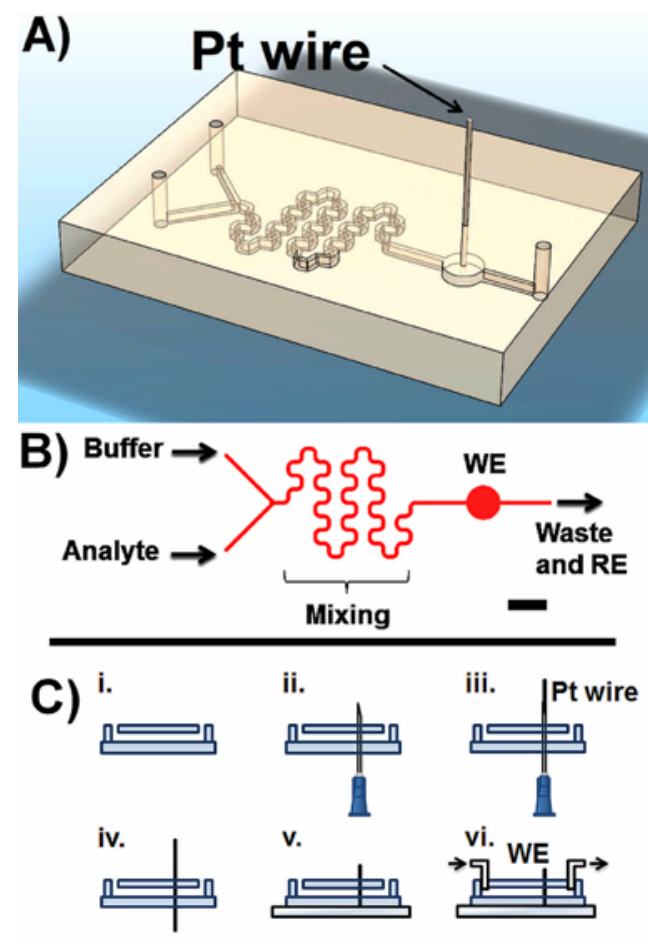

Figure 1: Design and characterization of the device. A) 3D schematic of the device, showing the Pt electrode inserted into the sensing area. B) Scheme of the device layout, showing the buffer and analyte inlets, the mixing serpentines, the sensing site with the working electrode (WE), and the waste outlet, where the reference electrode (RE) is placed (the black bar indicates $1 \mathrm{~mm}$ ). C) Scheme of the device fabrication, showing the insertion of the Pt wire electrode through a syringe needle.

To investigate this possibility, we compared the responses of an electrode incorporated into a fluidic channel to variations in analyte concentration. The outer-sphere redox probe ferrocenemethanol $(\mathrm{FcMeOH})^{20,23,24}$ was used, to disentangle our observations from secondary mechanisms that are typical of inner-sphere reactions (adsorption, formation of chemical bonds, changes in chemical structure, etc.), which may delay the response. This redox probe is hence expected to allow for the direct observation of the effect of convective and diffusive mass transport on the measured current profiles. Other chemicals could have been used, such as hexaamineruthenium (III). In the case of hexaamineruthenium (III), and because of the oxygen permeability of poly-dimethylsiloxane (PDMS), reduction oxygen at the electrode would have been hard to avoid during electroanalysis. This additional reaction would have complicated the analysis of our system. This justified the choice of $\mathrm{FcMeOH}$, to avoid interferences from background species. Two electrochemical detection schemes have been compared, AMP and CV at a relatively high scan rate $(S R)$ of $10 \mathrm{~V} \mathrm{~s}^{-1}$. Several concentration profiles (sawtooth, sine and step) have been tested, and different flow rates, $v$, were investigated (for a $4 \mathrm{~s}$ step concentration profile). In all the cases, the $\mathrm{CV}$ traces were found to be delayed in comparison to the AMP traces. This delay over the amperometric response time was found to increase with the flow velocity (in the case of a step concentration profile). Spectral analysis of the data showed that, above a specific flow velocity, the ability of CV to resolve the variations in concentration decreases in comparison to AMP. Finally, the effect of buffer viscosity was studied.

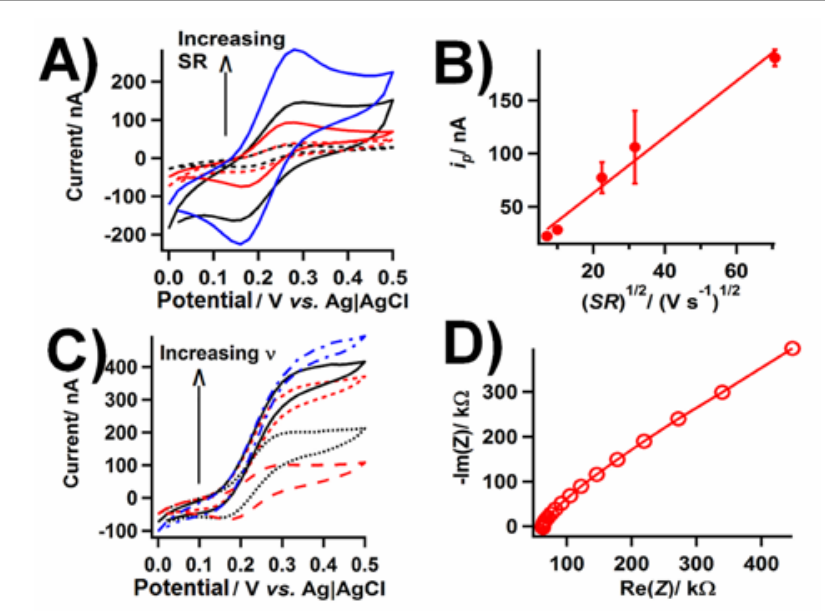

Figure 2: Electrochemical characterization of the system. A) Characteristic CV responses, for $1 \mathrm{mM} \mathrm{FCMeOH}$ in PBS, for different scan rate $S R(50,100,500$, 1000 and $5000 \mathrm{mV} \mathrm{s}^{-1}$ ). B) Dependence of the oxidative peak current $i_{p}$ with $S R^{1 / 2}$ determined from the curves in $A)(N=3$, average $\pm S D$, datasets obtained from 2 different chips). C) Characteristic CV responses for $1 \mathrm{mM} \mathrm{FCMeOH}$ in PBS, for different flow rate $v\left(0,2,10,20\right.$ and $\left.30 \mu \mathrm{s} \mathrm{s}^{-1}, S R=100 \mathrm{mV} \mathrm{s}^{-1}\right)$. D) Typical EIS trace obtained for a static solution of $1 \mathrm{mM} \mathrm{FCMeOH}$ in PBS, showing a clear diffusion-limited behavior for all the frequencies considered ( $10 \mathrm{~Hz}$ to $100 \mathrm{kHz}$ ).

\section{Results and discussion}

\section{Electrochemical characterization of the device}

Figure 1 shows the finished device, highlighting the position of the Pt wire used as a WE, as well as the fabrication procedure (see the Experimental section for details). To characterize the electrochemical properties of the system, the characteristics of $\mathrm{CV}$ performed in $1 \mathrm{mM}$ FcMeOH, in PBS, were analyzed (here, the $\mathrm{CV}$ were not background-subtracted). Figure 2A shows the impact of increases in $S R$ on the CV traces $(S R=50,100,500$, 1000 and $5000 \mathrm{mV} \mathrm{s}^{-1}$ ). At high $S R$, the system shows well defined peaks, and in all the traces, a clear increase in oxidative current is observed at a potential consistent with $\mathrm{FcMeOH}$ oxidation. ${ }^{25}$ Furthermore, the oxidative peak current $i_{p}$ increases with $S R$. The magnitude of $i_{p}$ is known to be proportional to the square root of $S R$, as it is directly related to the diffusion layer thickness. ${ }^{26-28}$ The graph presented in Figure 2B shows a clear linear relationship between $i_{p}$ and $S R^{1 / 2}$. Similarly, Figure 2C shows typical CV traces obtained for increasing $v(0,2,10,20$ and $30 \mathrm{\mu l} \mathrm{s}^{-1}$ ). As expected, an increase in $v$ results in a higher diffusion-limited current $i_{s s}$ (i.e. the current plateau corresponding to steady-state situated at voltages higher than the voltage at which $i_{p}$ occurred in Figure $2 \mathrm{~A}$, on the right side of the CV) because of the increased mass transport. Indeed, for these flow rates, mass transport is not solely controlled by local diffusion anymore, but principally by convection. Furthermore, as pointed out by several reports, for different electrode geometries, $i_{s s}$ increases linearly with $v^{1 / 3} \cdot{ }^{29-33}$ This dependence was also observed in our experimental analysis (data not shown). 

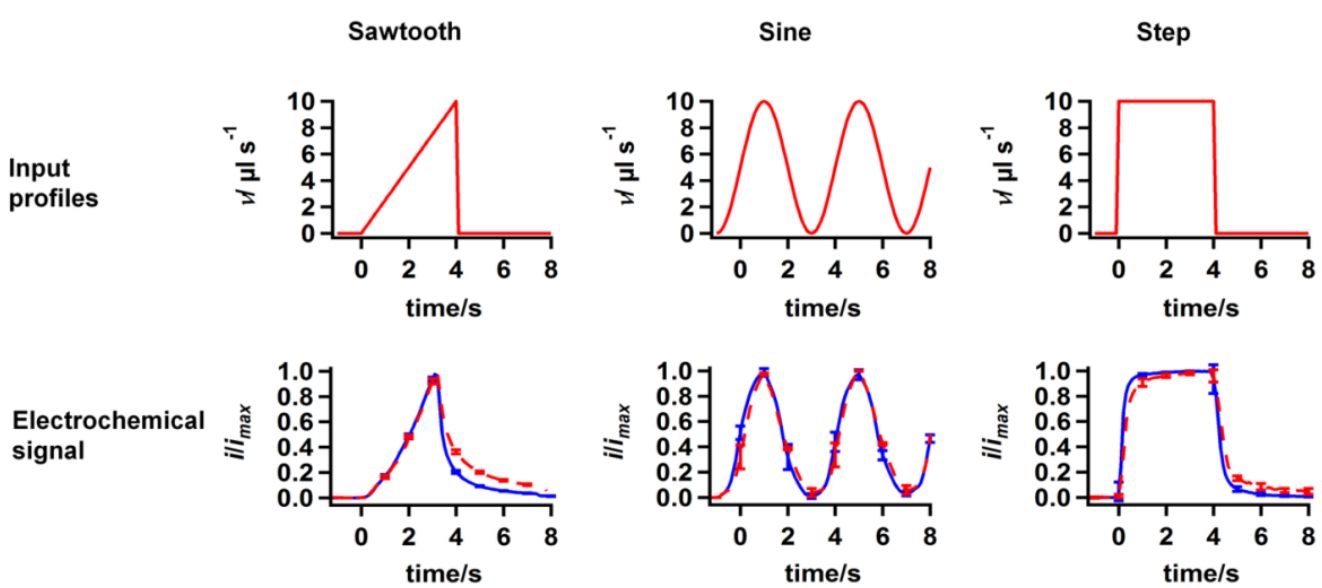

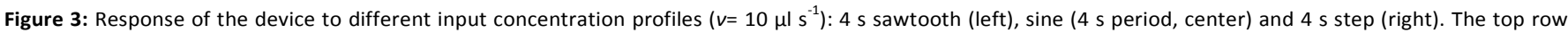

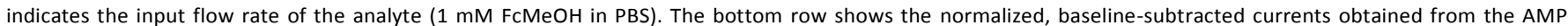
(solid line) and CV (dashed line) traces (from 3-4 cycles, average \pm SD).

Finally, to emphasize the outer-sphere nature of this redox reaction, EIS scans like the one shown in Figure $2 \mathrm{D}$ were performed. As expected, a linear-like graph, revealing a pure diffusion-limited behavior, is obtained. ${ }^{26,34}$ This indicates that the charge transfer resistance is low, and that the reaction is thermodynamically favored, i.e. that there are no secondary phenomena (formation of chemical bonds, adsorption, etc.) hindering the electron transfer. The traces were fitted with a simplified version of Randles' model, where a resistor modeling the resistance of the solution $R_{S}$ is connected in series with a Warburg impedance $Z_{W}$, accounting for the diffusion of the analytes. The modulus of the Warburg impedance was $\sqrt{2}\left(A_{W} / \sqrt{2 \pi f}\right)$, where $f$ is the applied frequency and $A_{W}=$ (2.94 \pm 0.07$) 10^{6} \Omega \mathrm{s}^{-1 / 2}(\mathrm{~N}=3)$. The solution resistance can be determined from Figure $2 \mathrm{D}$ as $54 \pm 8 \mathrm{k} \Omega(\mathrm{N}=3)$. This value can be understood by the presence of the connecting microchannel between the WE and the RE, which is filled with PBS. The conductivity of PBS is $1.5 \mathrm{mS} \mathrm{cm}^{-1}$, 35 while the channel separating the WE from the RE is $100 \mu \mathrm{m}$ high, 100 $\mu \mathrm{m}$ wide, and about $1 \mathrm{~mm}$ long, resulting in a calculated resistance of a few $100 \mathrm{k} \Omega$. This is higher than the measured value, but the presence of the $1 \mathrm{mM}$ FcMeOH was not taken into account in this calculation, and the precise geometry of this section of the chip is unknown, as the site of the hole punched for the connection cannot be very precisely controlled. However, the typical behavior of a charge transfer resistance in parallel with the electrode double layer capacitance (a semicircle starting at the origin in the plot of Figure 2D) is not observed in this EIS scan, up to $100 \mathrm{kHz}$. Overall, the increased solution resistance has no effect on the high-frequency response of our system. Hence, the time response of our device should mostly be described by the diffusive and chemical kinetics of the electrochemical reaction near the electrode, rather than be determined by microchannel design. This is further supported by calculating the electrode double layer capacitance from the CV data (see Supporting Information). This capacitance was evaluated as $3 \mathrm{nF}$. If this capacitance is in series with the solution resistance $R_{s}$, a characteristic time constant of $\sim 0.2 \mathrm{~ms}$ is expected. As detailed below, this time response is much faster than the expected typical time scale of the diffusive processes (about $10 \mathrm{~ms}$, see below the discussion on $\tau_{D}$ ), and can be neglected.
Furthermore, the curve of Figure 2D can be modeled, in a first approximation, by the following impedance $Z_{e q}$ $Z_{e q}=R_{s}+Z_{w}$

$$
=R_{s}+\frac{A_{w}}{\sqrt{2 \pi f}}+\frac{A_{w}}{j \sqrt{2 \pi f}}=\left(R_{s}+\frac{A_{w}}{\sqrt{2 \pi f}}\right)-j \frac{A_{w}}{\sqrt{2 \pi f}}
$$

with $A_{w}$ being the Warburg coefficient, defined as ${ }^{26,36}$

$A_{W}=R T / n^{2} F^{2} A C_{0} \sqrt{2 D}$

where $R$ is the gas constant, $T$ is the temperature, $n$ the number of electrons transferred, $F$ the Faraday constant, $A$ the electrode surface area, $D$ the diffusion coefficient and $C_{0}$ the bulk concentration of the analyte. The diffusion coefficient of $\mathrm{FcMeOH}$ in aqueous buffers was evaluated by others as $(7.6 \pm$ 0.4) $10^{-10} \mathrm{~m}^{2} \mathrm{~s}^{-1} \cdot{ }^{19}$ Hence, the theoretical value of $A_{w}$ was calculated for our experimental conditions as $4.310^{5} \Omega \mathrm{s}^{-1 / 2}$. This is lower than the experimental result, and is understood as an indication of hindrance in the diffusive dynamics of the reaction from the microchannels. Indeed, the low channel height $(100 \mu \mathrm{m})$ will constrain the mass transport at the extremities of the cylindrical electrode, thus reducing the expected current, in comparison to the same geometry in a free, unconstrained fluid, and leading to an apparent increase in diffusive resistance. This is supported by the scan rate analysis (Figure 2B). The slope of the fitting curve was extracted, and the expression of the peak current for a cylindrical electrode, provided by Matsuda et al. ${ }^{27}$ was used to evaluate the electrode surface area. The theoretical value was smaller than the one expected from our electrode dimensions, thus showing that restricted diffusion in this thin film of buffer leads to an apparent smaller electrode surface, and a larger $Z_{W}$.

\section{Potential scanning induces a delay}

The response of AMP and CV were initially compared by comparing the traces recorded during the injection of different concentration profiles of $\mathrm{FcMeOH}$, as shown on Figure 3. The maximum concentration was $1 \mathrm{mM}$, and $v$ was $10 \mu \mathrm{s} \mathrm{s}^{-1}$. Three different flow profiles were used, a sawtooth, a sine wave and a step, as shown on Figure 3, top. The analyte concentration was adjusted by modulating the ratio of the flow rates of the two input channels to the chip (buffer and analyte), the sum of the respective flow rates being fixed at $10 \mu \mathrm{l} \mathrm{s}^{-1}$. Note that the currents in Figure 3 are normalized by their maximum values 
$\left(i_{\max }\right)$; for $\mathrm{CV}$ measurements, $i_{\max }$ is of the order of $\sim 250 \mathrm{nA}$, while this is typically lower in AMP recordings, where one finds e.g. $\sim 100 \mathrm{nA}$, for $v=10 \mu \mathrm{l} \mathrm{s}^{-1}$.

The electrochemical response obtained at the WE was recorded and processed (normalization and background subtraction), as detailed in the Experimental Section. The averaged traces $(\mathrm{N}=$ $3-4 ; \pm$ SD) are presented in Figure 3, bottom. To facilitate the comparison, the traces were overlaid and aligned. In the case of the sawtooth and step profiles, this was done by aligning the initial rises in current, indicating the onset of the increase in FcMeOH concentration. For the sine curve, the maximum currents, indicating the top of the concentration wave, were aligned. In all the considered cases, the CV measurements appear to be delayed, in comparison to the AMP traces.

A closer inspection indicates that this delay is more prevalent for fast variations in FcMeOH concentration. For instance, for the sine wave, and the rising part of the sawtooth, the AMP and $\mathrm{CV}$ traces are largely or totally overlapping. However, when the concentration changes rapidly, as seen for the drop at the end of the rise of the sawtooth, or in the case of the step, the $\mathrm{CV}$ response is largely delayed in comparison to the AMP traces. Overall, the difference between $\mathrm{CV}$ and AMP becomes more marked with fast, steep changes in concentration. In the sine waves, the changes are much smoother than for the step or the sawtooth, the delayed response of $\mathrm{CV}$ being hence much smaller than for the step or sawtooth profiles.

As an outer-sphere redox couple was used in our analysis, and the EIS traces show a diffusion-limited behavior, even at high frequencies, the recorded current should be largely controlled by the ionic mass transport of the analyte of interest. Because of the nature of voltammetric sensing, where the specificity of the measured signal principally arises from the stabilization of the diffusion layer under the depletion of the analyte of interest at the vicinity of the electrode surface, this observation strongly supports that the observed delay stems from the hindered dynamics of the depletion layer during the repeated voltammetric sweeps. This hypothesis is tested by modifying the properties of the flow, mostly the velocity and the viscosity of the buffer (vide infra).

\section{Effect of flow velocity}

In this section, four different levels for $v$ were tested $\left(10 \mu \mathrm{s} \mathrm{s}^{-1}\right.$, $\left.5 \mu 1 \mathrm{~s}^{-1}, 1 \mu \mathrm{l} \mathrm{s}{ }^{-1}, 0.5 \mu 1 \mathrm{~s}^{-1}\right)$. Step-shaped concentration profiles were used, injecting a $1 \mathrm{mM}$ FcMeOH solution for $4 \mathrm{~s}$. As seen on Figure 3, for the step profile, the responses of the system to the onset and the end of the step are qualitatively different. To simplify the analysis, we here focus on the current profiles induced by the rising section of the step (i.e., from 0 to $4 \mathrm{~s}$ ). The results obtained for the different $v$ values, for the AMP and CV methods, are shown in Figure 4A-D.

In general, the current traces, both for $\mathrm{CV}$ and AMP, get sharper as $v$ increases. This behavior can be explained by the decreased time during which dispersion of the analyte at the edge of the injected plug occurs for higher velocities, leading to less broadening of the ascending slope of the concentration profile. The effects of fluid viscosity, known as Taylor dispersion, tend to amplify this phenomenon. ${ }^{37}$ The ascending sections of the traces shown on Figure 4A-D were well-fit with the positive part (i.e. for $\mathrm{t} \geq 0 \mathrm{~s}$ ) of the error function erf

current $(t)=\alpha \operatorname{erf}\left(\frac{t}{\sigma \sqrt{2}}\right)$

$=\alpha \frac{2}{\sqrt{\pi}} \int_{0}^{\frac{t}{\sigma \sqrt{2}}} e^{-u^{2}} d u$

where $\alpha$ is a fitting parameter. The parameter $\sigma$ is the standard deviation of the Gaussian integrated in the definition of the error function. This function is known to describe the time variations, under the effects of diffusion, of the concentration profile between two regions of different concentrations. ${ }^{37}$ This phenomenon is similar to the Taylor dispersion (vide infra).
A) $v=10 \mu \mathrm{s} \mathrm{s}^{-1}$

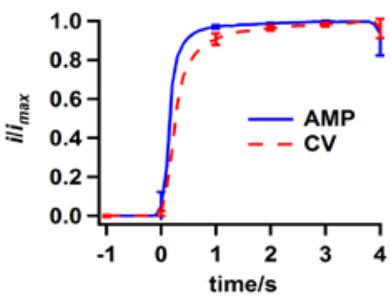

D) $v=0.5 \mu \mathrm{s} \mathrm{s}^{-1}$

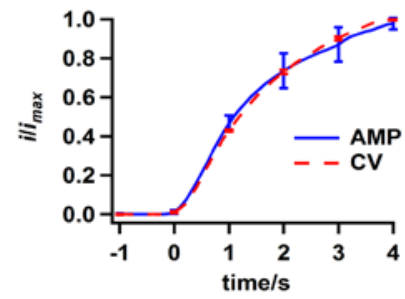

B) $v=5 \mu / \mathrm{s}^{-1}$

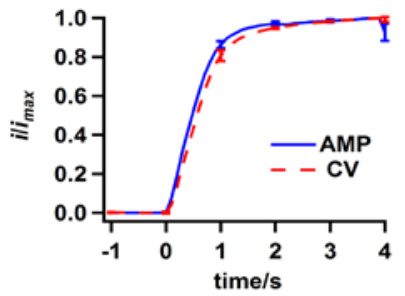

E) $\sigma_{A M P}$ and $\sigma_{C V} v s . V^{-1 / 2}$

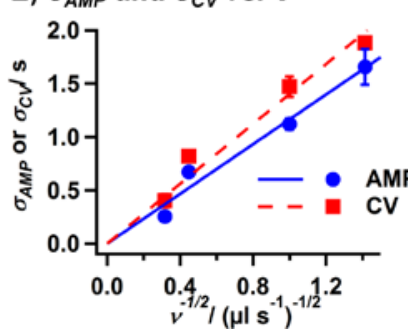

C) $v=1 \mu \mathrm{s} \mathrm{s}$

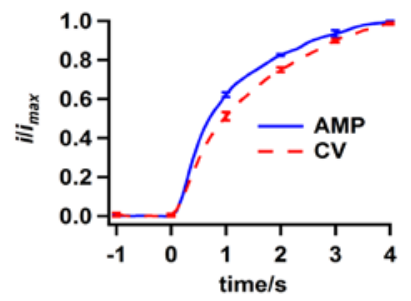

F) Model of the system

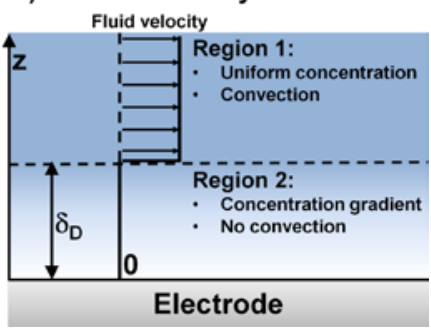

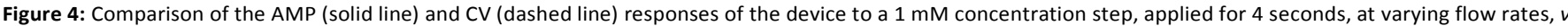

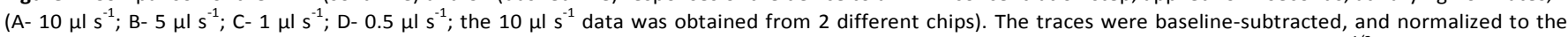

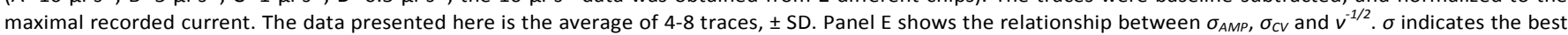

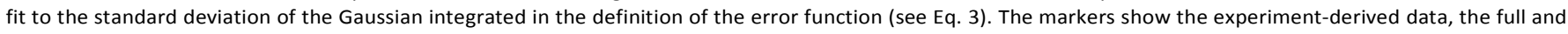

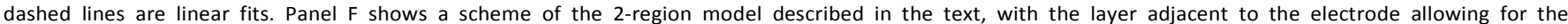

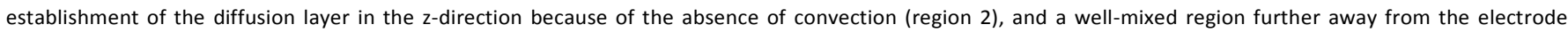

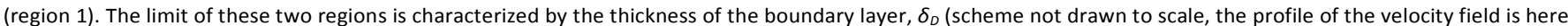
non-significant; the arrows indicate the magnitude of fluid velocity, the color of the background the concentration gradient). 
However, because of the fast current rise at $\mathrm{t}=0 \mathrm{~s}$ induced by the initial large flow-induced mass transport of analyte, only the right part of the erf function is considered. After this sharp onset, the rising section is largely induced by dispersion at the edge of a plug of analyte. Hence, the positive part of erf is a well-chosen model of the observed current traces, and was found to fit well with the experimental data. This allows for a close description of the sharp current increase as the bolus on analyte encounters the electrode, as well as the dispersioninduced broadening of the data characterized by $\sigma$.

The experimental values obtained for $\sigma$ are plotted in Figure 4E as a function of $v^{-1 / 2}$ (vide infra), and are also reported in Table S1 (see Supporting Information), where $\sigma_{C V}$ and $\sigma_{A M P}$ conveniently indicate the values obtained from the $\mathrm{CV}$ and AMP traces, respectively. The values for $\sigma_{A M P}$ are significantly smaller than the ones for $\sigma_{C V}$, for $v \geq 1 \mu \mathrm{s} \mathrm{s}^{-1}$, indicating a faster current rise, in good agreement with the slower current variations of the $\mathrm{CV}$ analysis observed on the experimental traces of Figure 4. No significant difference is observed for $v=$ $0.5 \mu \mathrm{s} \mathrm{s}^{-1}$ (most right point in Figure 4E), thus supporting the hypothesis that the delayed response of $\mathrm{CV}$ is more important for fast increases in concentrations, i.e. for fast transients.

The characteristic rise times $\sigma_{C V}$ and $\sigma_{A M P}$ are both indicative of the level of dispersion in the system, as they are related to the steepness of the current rise, and can be quantitatively related to $v$. The Péclet number $P e=V_{0} d / D$ where $d$ is the typical dimension of the channel, radial to the flow (here, $100 \mu \mathrm{m}$ ) and $V_{0}$ is the fluid velocity at the center of the channel, indicates whether convection or diffusion dominates in the considered mass transport phenomena. ${ }^{37}$ For high $P e$, the system is convection-controlled. In our device, for the range of velocities considered, $P e>5000$. As a consequence, the steepness of the ramp at the buffer/ analyte interface mostly arises from Taylor dispersion, rather than from Brownian motion. Even though the case of a rectangular channel has been detailed in the literature, ${ }^{38}$ we use here for the sake of simplicity the expressions derived for a circular channel, of radius $r$, the two cases differing by a scaling factor depending on the dimensions of the channel. ${ }^{39}$ In this case, it is possible to define a dispersion length $w_{d}$, characterizing the Taylor dispersion at the analyte/ buffer interface after a time $t,{ }^{37}$ while $t$ can also be written as $l_{\text {channel }} / V_{0}$, where $l_{\text {channel }}$ is the length of the channel:

$$
\begin{aligned}
& w_{d}=r V_{0} \sqrt{\frac{48 t}{D}} \\
& \propto \sqrt{V_{0}} \\
& \propto \sqrt{v}
\end{aligned}
$$

Note that, because of the convection control observed in our system, the diffusion-induced broadening of the ascending concentration of a plug injected in the microchannel (here indicated by $D$ ) is quantitatively different from a diffusion profile considered in the usual equation that describes the diffusion length as $L_{D}=\sqrt{2 D t}$. The distance $w_{d}$ can be related to the time dimension, and hence to the characteristic times $\sigma_{C V}$ and $\sigma_{A M P}$, by dividing Eq. 4 by $v$ :

$\sigma_{A M P}$ or $\sigma_{C V} \propto \frac{1}{\sqrt{v}}$

Based on this analysis, the experimental values were represented on Figure $4 \mathrm{E}$ as a function of $v^{-1 / 2}$. A clear linear relationship can be observed, demonstrating that the variations in the steepness of the current rises are mostly controlled by the dispersion of the analytes. However, this equation is expected to poorly describe the system as $v^{-1 / 2}$ increases (or $v$ decreases), leading to lower $P e$ values and the system would become diffusion-controlled.

A common approximation in hydrodynamic techniques states that the concentration of analyte is maintained uniform, and equal to its bulk concentration, beyond a certain distance $\delta_{D}$ from the electrode (Figure 4F). ${ }^{26}$ Below this distance, there is no convective movement, allowing the formation of the diffusion layer. This is somewhat similar to Prandtl's boundary layer concept, where friction exerted on an object in a convective flow only arises from the fluid immediately adjacent to the surface of the object. ${ }^{40}$ Using this approximation, it is therefore possible to model the system in two regions, characterized by their normal distance $z$ from the electrode surface. This model therefore consists in discretizing the system in two regions, assuming that in the area sufficiently close to the electrode, the effect of convection can be neglected and the mass transport is diffusion-controlled. A first region (Region 1 on Figure 4F) is identified for $z>\delta_{D}$, where convection is observed, and the concentration is uniform. Using the characteristic diffusion-limited regime of AMP, we assume that the AMP traces actually describe the averaged current at the vicinity of the electrode, in this region. The second region (Region 2 on Figure 4F) is the diffusion layer, for $0 \leq z \leq \delta_{D}$, and no convection occurs in this layer, following Prandtl's hypothesis. In the case of AMP, this region can be considered as stable, with the bulk concentration of the analyte varying at its outer limit, hence changing the diffusion-limited current. However, in the case of $\mathrm{CV}$, this region is not stable, and its thickness varies constantly as the electrode potential is swept back and forth. The characteristic time $\tau_{D}$ for the diffusional relaxation of the concentration boundary layer has to be taken into account. This time was previously used to quantify the ability of the diffusion processes observed at the electrode to respond to alterations in mass transport at one edge of the diffusion layer after variations of the flow velocity. ${ }^{41,42}$ By analogy with these works, the reaction rate, controlled by the potential ramps during $\mathrm{CV}$, will also alter the mass transport defining the diffusion layer, and the time required for the diffusion layer to adapt to these new conditions is expected to be described by $\tau_{D}$. This characteristic time can be related to $\delta_{D}$ : 41,42

$\tau_{D}=\frac{\delta_{D}{ }^{2}}{2 D}=\frac{D}{2 k_{t}{ }^{2}}$

where $k_{t}$ is the mass transport coefficient of the electrode, defined as

$k_{t}=\frac{i_{s s}}{n F A C_{0}} \propto v^{1 / 3}$

as $i_{s s}$ is proportional to $v^{1 / 3} \cdot{ }^{29-33}$ The expression presented in Eq. 6 shows that $\tau_{D}$ is intuitively the diffusion time of an analyte across the diffusion layer, i.e. the time required for the information to diffuse across this layer. Interestingly, it can also be shown that $\tau_{D}$ is analogous to an RC relaxation time, as detailed in the Supporting Information. This analogy further hints that the intrinsic dynamics of the diffusion layer may act as an RC circuit, dampening the response of the system when exposed to fast concentration transients. This analysis also shows that the geometry of the system will only alter the response of the system by modifying the rate of change in analyte concentration in the electrode vicinity, mostly via Taylor dispersion. The actual response of the system, from which the delayed response of CV arises, is controlled by the dynamics of Region 2. 
By combining Eq. 6 and 7, we can also conclude that $\tau_{D}$ and $\delta_{D}$ decrease for higher $v$, as the higher velocity-induced mass transport overcomes analyte diffusion. Using the experimental $i_{s s}$ values, obtained from the plateau-like regions on the right of the CVs presented in Figure 2C for different values of $v, \tau_{D}$ was found to be in the 1-100 ms range for the velocities considered in the experiments $(110,24,8,5$ and $5 \mathrm{~ms}$ for flow rates of 0,2 , 10,20 and $30 \mu \mathrm{l} \mathrm{s}^{-1}$, respectively).

These numerical results are of the order of the time constant predicted from the Warburg impedance analysis from the EIS data when $v=0 \mu \mathrm{s} \mathrm{s}^{-1}$. Indeed, the Warburg element provides a phenomenological description of the effect of diffusion on the measured current near an electrode for a frequency of interest. From EIS curves like presented in Figure 2D, and by analogy with the impedance of a capacitor, we define from the imaginary part of this equation a capacitance $C_{W}=$ $1 /\left(A_{W} \sqrt{2 \pi f}\right)$, Eq. 1 can be simplified as

$Z_{e q}=\left(R_{s}+\frac{A_{w}}{\sqrt{2 \pi f}}\right)-j \frac{1}{2 \pi f C_{W}}$

Using an RC system, it is possible to define a characteristic time $\tau_{R C}$ from the impedance given in Eq. 8:

$\tau_{R C}=\left(R_{s}+\frac{A_{w}}{\sqrt{2 \pi f}}\right) C_{W}$

This time is expected to account for the response time of the system at a given frequency, the response being delayed by the diffusive processes occurring in the vicinity of the electrode.

For the $\mathrm{CV}$, the input frequency is typically the frequency of the triangular potential waveform, hence $10 \mathrm{~Hz}$ in our case. Using the experimental values reported above for the impedance, the characteristic time $\tau_{R C}$ is evaluated as $18 \mathrm{~ms}$ for a sweep frequency of $10 \mathrm{~Hz}$. This value is hence indicative of the characteristic diffusive time expected to control the system for this sweep frequency: the diffusion of species is faster than the rate of change of the voltage in our CV experiments, but will provide a delayed response. One should note that this fact holds also for higher frequencies, $\tau_{\mathrm{RC}}$ decreases with frequency, because the Warburg impedance decreases with frequency.

This analysis further stresses the impact of the intrinsic inertia of the diffusion layer dynamics in the control of the $\mathrm{CV}$ response. The effect of this relaxation time can explain the delayed response of $\mathrm{CV}$, as the diffusion layer has to be constantly re-established. Because of the steady-state, diffusion-limited regime of the AMP analysis, we can formulate the hypothesis that the AMP traces give a direct recording of the concentration profiles at the vicinity of the electrode. The initial current increase is mostly indicative of Taylor dispersion of the plug in the channels, before the analyte reaches the sensing site. In the case of $\mathrm{CV}$, the current rise is also largely controlled by dispersion (see Figure 4E), but an additional diffusion-related factor induces the delayed response observed in Figures 3 and 4.

Altogether, both the analyses on the Warburg impedance and the relaxation of the diffusion layer indicate that the cyclic variations in diffusion layer geometry encountered in $\mathrm{CV}$ results, both qualitatively and quantitatively, in a RC-type component, dampening the response kinetics of the system to concentration variations.

\section{Frequency response of the fluidic/ electrochemical system}

The fact that the voltammetric response is delayed over amperometry for $v \geq 1 \mu \mathrm{l} \mathrm{s}^{-1}$, but not for $v=0.5 \mu \mathrm{l} \mathrm{s}^{-1}$ suggests an investigation of the frequency dependence of the electrochemical signal. More specifically, following an analogy with the mechanisms underlying the formation of an image in an optical device, as suggested by others, ${ }^{22}$ the final electrochemical signal $S$ recorded as a function of time by the device, can be obtained from the convolution of the input concentration profile $C$ (in our case, for example, a 4 s step profile) with the impulse function $I F$, a characteristic feature of the device: ${ }^{43,44}$

$S(t)=\int I F\left(t-t^{\prime}\right) C\left(t^{\prime}\right) d t^{\prime}$

$\equiv(I F * C)(t)$

with * defining the convolution operator. The function IF here principally accounts for the degradation of the signal induced by Taylor dispersion, delayed response of the electrochemical sensing, etc., and therefore the response of the total fluidic/electrochemical system is not solely determined by the sensing principle. Analyzing IF is hence important to understand the behavior of the device.
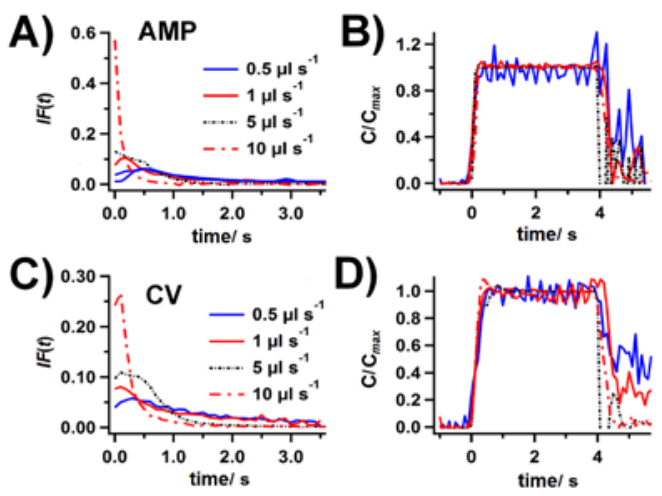

D)
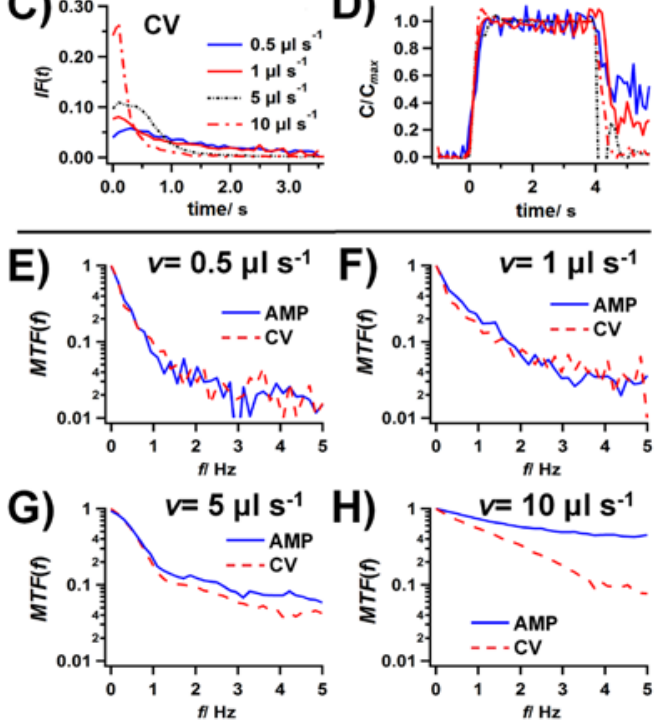

Figure 5: Impulse function (IF) for A) AMP and C) CV responses for varying flow rates, $v$. The corresponding deconvoluted concentration traces are shown on $B$ ) for $A M P$ and $D$ ) for $C V$ (the color codes are the same as the ones of panels $A$ and B). The IF was used to compute the MTF (E- $0.5 \mu \mathrm{s} \mathrm{s}^{-1} ; \mathrm{F}-1 \mu \mathrm{l} \mathrm{s}$; G- $5 \mu \mathrm{l} \mathrm{s}{ }^{-1} ; \mathrm{H}-10$ $\mu \mathrm{s} \mathrm{s}^{-1}$ ) for AMP (solid line) and CV (dashed line).

The Dirac function $\delta$ is defined as the function returning 0 for $t \neq 0 \mathrm{~s}$, and whose integral is 1 over $\mathbb{R}$. More intuitively, $\delta$ can be approximated as an infinitely high, infinitely sharp peak centered over $0 \mathrm{~s}$, the function being otherwise equal to 0 . Interestingly, $\delta$ is the neutral element of the convolution, and IF could theoretically be obtained by injecting a narrow, highly concentrated step of analyte. This is analogous to obtaining the point spread function (PSF) for an optical system. ${ }^{44,45}$ However, this would be experimentally hard to achieve, because of the high pressures and fast actuation required. Furthermore, the result would only be an approximation of $\delta$. A more elegant way is to consider the time integral of $\delta$, hence the Heavyside function $H,{ }^{22}$ leading to 
$I F(t)=(I F * \delta)(t)$

$=\frac{d}{d t}(I F * H)(t)$

The IF function can hence be obtained by differentiating the response of the device to a step of concentration. To this purpose, the experimental curves shown on Figure 4A-D were differentiated and normalized to their integral and are shown on Figure 5A and C, for the AMP and CV modes, respectively, for the different $v$ values. As expected from Eq. 3, the IF function shows a decay, initiated at $t=0 \mathrm{~s}$, as already reported by others (the function is set to 0 for $t<0 \mathrm{~s}$ ). ${ }^{22}$ The profiles of the IF are similar to the right part of a Gaussian curve, in good agreement with Eq. 3. In our analysis, only the decaying section is expected to be significant. Applying this procedure implies that the calculated IF functions contain all broadening and dispersion effects, while the concentration profiles of Eq. 10 are considered 'ideal' step functions. The validity of our approach was further confirmed by numerically deconvoluting these IF functions from the signal function $S(t)$ (see Eq. 10) to retrieve these 'ideal' concentration profiles. The results are shown on Figure 5B and D: the profiles are well-defined step functions indeed, especially for the rising part of the curves, for both AMP and CV recordings.

As stated above (see Eq. 3), the width of the $I F$ is related to the slope of the curves shown on Figure 4, and hence to the Taylor dispersion. As expected, especially from the fitting to the error function and Figure $4 \mathrm{E}$, the $I F$ get sharper as $v$ increases, thus indicating a better time resolution, for both AMP and CV. Also, comparison of the IF obtained for AMP to the ones obtained for $\mathrm{CV}$ reveals that the AMP ones appear to be sharper, hence inducing a faster response and better temporal resolution. Again, this was expected, as $\sigma_{A M P}<\sigma_{C V}$ for all the $v$ values considered.

Further analysis of the delayed response of CV over AMP can be obtained by considering the Fourier transform on the $I F$, and especially the modulation transfer function $(M T F)$, defined as the modulus of the Fourier transform of $I F^{44,46-48}$

$\operatorname{MTF}(f) \equiv|\mathcal{F}(I F)|(f)$

where $\mathcal{F}$ is the Fourier transform operator, and $f$ is the frequency. The MTF therefore indicates how the amplitude of the output signal is modulated with frequency. Experimentally, the MTF can also be calculated using the concept of contrast

contrast $=\frac{i_{\max }-i_{\min }}{i_{\max }+i_{\min }}$

where $i_{\min }$ and $i_{\max }$ are the minimum and maximum signal intensity. The $M T F$ is then the ratio of the output contrast contrast $_{\text {output }}$ to the input contrast contrast $_{\text {input }}{ }^{44}$ as a function of $f$

$\operatorname{MTF}(f)=\frac{\text { contrast }_{\text {output }}(f)}{\text { contrast }_{\text {input }}(f)}$

The MTFs were computed, by taking the amplitude of the fast Fourier transform of the $I F$, and are presented on Figure $5 E-H$, for different values of $v$. Generally, the MTF decreases with increasing $f$. This is consistent with the fact that high-frequency information tends to be lost because of dispersion, sampling, smoothing, etc. The steepness of this decrease is less important for higher values of $v$. Indeed, the concentration profiles are sharper at high flow rates, because of the decreased contribution of Taylor dispersion, see Eq. 5. For $\nu=0.5$ or $1 \mu 1 \mathrm{~s}^{-}$ ${ }^{1}$, the MTF for the AMP and CV methods are largely similar. At $5 \mu \mathrm{s}^{-1}$, there is still a good overlap at low frequencies, but as $f$ increases above $1 \mathrm{~Hz}$, the AMP seems to offer a better contrast, as its MTF is above the one of CV. Finally, at $10 \mu \mathrm{s} \mathrm{s}^{-1}$, the $M T F$ of AMP is above the one of $\mathrm{CV}$ for all the frequencies considered, showing that AMP better resolves signal variations, and that much less information is lost.

As detailed in Eq. 14, the MTF can also be obtained directly by recording the response of the device to a series of sine waves, similar to the one shown on Figure S3 (see in the Supporting Information), of decreasing periods (5, 4, 3, 2 and 1 s). The resulting traces are shown on Figure S3A, and the output contrast was calculated from this trace, for at least 6 cycles. The contrast of the input signal, contrast $t_{\text {input }}$ in Eq. 14, was here taken as 1 , with the concentration of analyte varying from 0 to $1 \mathrm{mM}$. The flow velocity was set at $5 \mu \mathrm{s} \mathrm{s}^{-1}$, as this value seemed to be a limiting value, where the MTF starts to be different for AMP and CV, as was clear from previous analysis. The obtained values are summarized on Figure S3B. For all the considered frequencies, the AMP showed a significantly ( $\mathrm{p}<$ 0.001) higher $M T F$, and hence a better transmission of the signal. This difference was not distinguishable on the MTF computed from the $I F$, but the shapes of the curves are very similar over the frequency range considered. This further establishes the robustness and validity of the MTF approach, in addition to its spectral nature, providing more information on the step response of the device.

\section{Variations of fluid viscosity}

The previous discussion emphasized the role of the relaxation of the diffusion layer in the delayed response of $\mathrm{CV}$. To further investigate this phenomenon, it is possible to adjust some of the diffusion-relevant parameters. In this perspective, the diffusion coefficient of FcMeOH was changed by modifying the viscosity of the buffer by adding glycerol to the PBS. ${ }^{49-51}$ Volume fractions of glycerol of $10 \%$ and $20 \%$ were used. The same mixture was used for the background buffer and the preparation of the FcMeOH solution, to avoid gradients of glycerol concentration between the different solutions. The viscosities $\eta$ of the different PBS/ glycerol mixtures used in the experiments were interpolated from the values reported in ref. 52 (0\%: 1.01 ср; 10\%: 1.45 ср; 20\%: 2.18 ср). The diffusion coefficient can be related to $\eta$ with $^{53}$

$D=\frac{k T}{6 \pi \eta r_{p}}$

with $k$ the Boltzmann constant, and $r_{p}$ the hydrodynamic radius of the particle. Hence, in the $20 \%$ mixture, $D$ is half the one in PBS. The flow rate was $10 \mu \mathrm{s} \mathrm{s}^{-1}$, as fast flow rates emphasized the differences between AMP and CV. The obtained traces, and the corresponding data processing, are shown on Figure 6.

Here again, the $\mathrm{CV}$ traces were found to be delayed, in comparison to the AMP curves (Figure 6A-C). The IF and MTF analysis was here repeated. The IF were computed, and are shown in Figure 6D and E, for the AMP and CV traces, respectively. Interestingly, the $I F$ for the $\mathrm{CV}$ are unaltered by the variations of $D$ and $\eta$. However, the IF for AMP becomes broader, as the fraction of glycerol increases. This is in good agreement with Eq. 4. As the AMP responses to the concentration steps are well-described by the positive half of the error function, the IF obtained for AMP were fit with a Gaussian, centered over the maximum of the IF. The $\sigma$ values of the fitted Gaussians were $0.07 \mathrm{~s}, 0.09 \mathrm{~s}$ and $0.11 \mathrm{~s}$, for the $0 \%, 10 \%$ and $20 \%$ mixtures, respectively. Interestingly, the variations of these values are proportional to $D^{-1 / 2}$, in good agreement with the behavior expected from Eq. 4. Hence, the 

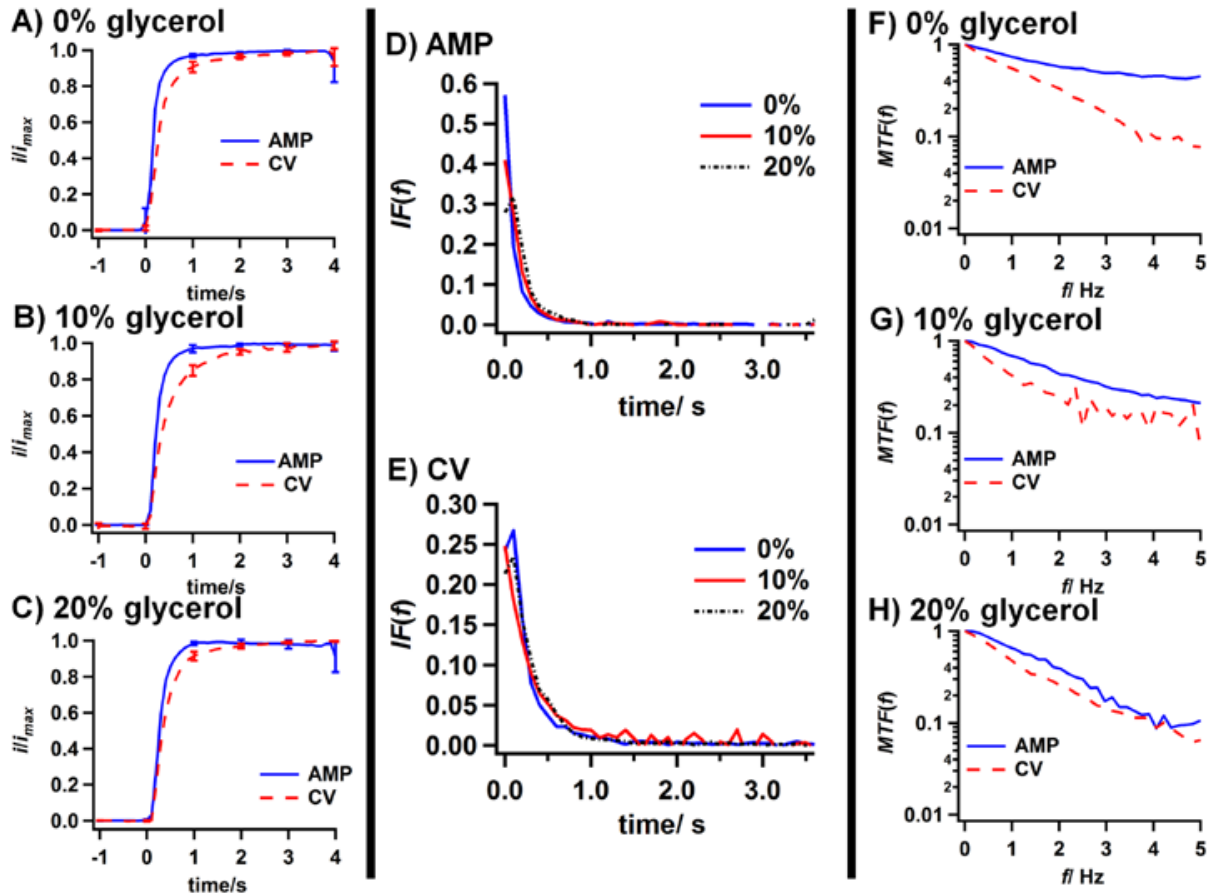

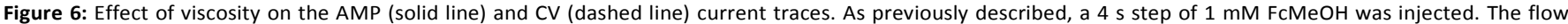

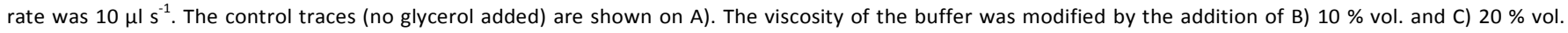

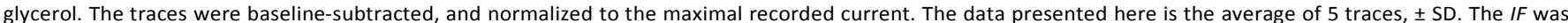

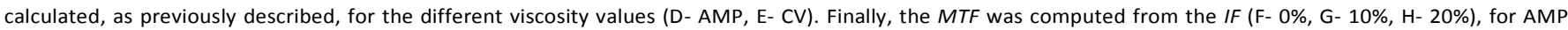
(solid line) and CV (dashed line).

increasing steepness in the decrease of the MTF (Figure 6F-H), for the AMP curves, can be explained by the broadening of the edge of the injected plug of analyte, hence reducing the temporal resolution of the device. This observation also further emphasizes the improved reliability of the $I F$ and MTF analysis over the observation of the unprocessed traces in the time domain, the apparent AMP traces looking similar for all the considered viscosities.

On the other hand, for the $\mathrm{CV}$, the $I F$ do not vary much and are all largely comparable for the different glycerol fractions. This is understood as further evidence of the filtering effect of $\tau_{D}$ over the observed concentration transients. This is also confirmed by the shape of the MTF, which is stable for increasing fractions of glycerol. Hence, CV is largely incapable of differentiating minute changes in concentration profiles induced by variations in viscosity, in comparison to AMP. However, closer comparison of the MTF obtained, for $\mathrm{CV}$, for $0 \%$ and $20 \%$ glycerol reveals that the slope of the MTF is slightly steeper at high glycerol fraction. As $i_{s s}$ is typically proportional to $D^{2 / 3}$ for a range of electrode geometries, $\tau_{D}$ can be expected to be proportional to $D^{-1 / 3}$ (Eq. 6 and 7 ). The characteristic frequency of the system associated to $\tau_{D}$ is therefore proportional to $D^{1 / 3}$. Hence, if we consider, for instance, $f_{25 \%}$, i.e. the frequency where the $M T F$ is 0.25 , we should expect a linear dependence with $D^{1 / 3}$ from Eq. 6 and 7 , corresponding to, if the $0 \%$ and $20 \%$ glycerol mixtures are considered, a decrease of $23 \%$. In good agreement with this consideration, the $f_{25 \%}$ were measured as $2.50 \mathrm{~Hz}$ and $2.03 \mathrm{~Hz}$, for the $0 \%$ and $20 \%$ glycerol mixtures, respectively. This further supports that the frequency response of the CV curves is largely controlled by the dynamics of the diffusion layer.

\section{Conclusions}

A microdevice, featuring a Pt microelectrode, was used to compare the time response of AMP and CV to different concentration profiles of the outer-sphere redox probe $\mathrm{FcMeOH}$. In all the considered experimental conditions, the $\mathrm{CV}$ traces were always found to be delayed in comparison to AMP. The delayed response of CV was previously reported, for neurotransmitters, and mostly attributed to adsorption of the analyte to the electrode surface. ${ }^{21,22}$ By using an outer-sphere redox couple, we show that mass transport also significantly delays the response of $\mathrm{CV}$. Analyte adsorption, in the case of an inner-sphere electron transfer, was not considered in our analysis, and the respective contribution of this phenomenon and mass transport cannot yet be elucidated. However, using different electrode materials and comparing the response of a reaction where surface adsorption is involved would be a way to investigate the surface affinity of the molecules. The effect of mass transport would have to be considered to evaluate precisely the mechanisms of analyte adsorption. Overall, the analysis of the system shows that the AMP traces are mostly controlled by the actual concentration profile, thus achieving high temporal resolution, when the CV traces are delayed by the relaxation of the diffusion layer induced by the repeated scanning of the electrode potential.

Electrochemical methods being increasingly used for highfrequency sensing, this finding could contribute to improving the temporal resolution of electrochemical methods, especially in the case of microfluidic channels. Only convective mass transport was considered in our analysis, but the competition between the variations of the diffusion layer and a large, time- 
varying diffusional gradient could potentially lead to the same observation. This fact is hinted by previous studies of exocytotic release, showing that the events recorded with $\mathrm{CV}$ were broadened in comparison to AMP. ${ }^{54}$ Furthermore, the presence of various matrices, gels and colloids in biological samples tend to induce different diffusion coefficients at the cell, tissue and organ levels. ${ }^{55-57}$ However, the glycerol analysis shows that $\mathrm{CV}$ may not be able to discriminate these different levels of diffusivity. Altogether, despite the improved chemical resolution of CV, AMP should be preferred when high temporal resolution is needed.

\section{Experimental}

\section{Chemicals}

FcMeOH and glycerol were both obtained from Sigma-Aldrich, Switzerland. Phosphate buffered saline (PBS, $\mathrm{pH}=7.4$ ) was obtained from PAA Laboratories GmbH. PDMS was obtained from Dow Corning, USA.

\section{Design of the device}

The layout of the chip was initially designed in Clewin 4, and is shown in Figure 1A, B. It featured two inlet channels on the left (width $=100 \mu \mathrm{m}$ ), for the buffer and the analyte streams. The junction of these channels was followed by several serpentines, for the convective mixing of the chemicals injected into the chip. Finally, the main channel reached the $1 \mathrm{~mm}$ diameter cylindrical sensing chamber. A waste channel was attached to the sensing chamber, for the evacuation of the reactants.

\section{Microfabrication of the device}

The chip was fabricated from PDMS using standard soft lithographic techniques. Briefly, a $100 \mu \mathrm{m}$ thick SU-8 photoresist layer was spin-coated on a clean Si wafer, and patterned through a $\mathrm{Cr}$ mask. After developing and silane functionalization of the SU-8 master, a 10:1 PDMS mixture was poured on the SU-8 structure and polymerized in a convection oven at $100{ }^{\circ} \mathrm{C}$ for $1 \mathrm{~h}$. To close the channels, the resulting patterned PDMS layer was bound to a flat PDMS surface, after surface-activation with air plasma (12 W for 70 s).

To integrate the Pt electrode inside the channel, a 29 G syringe needle was inserted across the sensing site through the PDMS layers, under microscopic observation (Figure 1C, steps i. and ii.). A Ø $51 \mu \mathrm{m}$ Pt wire (Science Products AG, Switzerland) was threaded in the lumen of the needle (step iii.). The needle was then carefully pulled out from the device (step iv), and the Pt wire was secured in place with PDMS. The finished device was then glued to a microscope slide with PDMS (step v.) to facilitate the handling of the system. Fluid connections were completed by inserting tubings into the port holes (step vi.).

The fluid flows were actuated from a computer-controlled Nemesys system (Cetoni GmbH, Germany) featuring two low pressure pump modules. The time base of the fluidic actuation was $0.1 \mathrm{~s}$. As detailed in the text, different time-dependent flow profiles (sine, step or sawtooth) were injected into the system, at different flow rates, $v$.

\section{Electrochemical measurements}

All the experiments were carried out with an Ivium Powerstat (Ivium, The Netherlands) functioning in the 2-electrode mode. The electrochemical system was completed with an $\mathrm{Ag} \mid \mathrm{AgCl}, 3$ $\mathrm{M} \mathrm{KCl}$, reference electrode (RE, Bioanalytical Systems Inc.,
USA). This electrode was placed in a $1 \mathrm{ml}$ pipette tip inserted in the outlet port hole, thus facilitating the connection of the RE to the rest of the system.

Unless stated otherwise in the text, the $\mathrm{CV}$ traces were recorded at $10 \mathrm{~V} \mathrm{~s}^{-1}$ between 0 and $0.5 \mathrm{~V}$ vs. Ag|AgCl. The currents corresponding to the oxidative peak were then analyzed, corresponding to $10 \mathrm{~Hz}$ sampling. The background current was obtained from blank traces performed in PBS, in similar experimental conditions, and subtracted from the traces to obtain a flat baseline (see Supporting Information).

The AMP traces were all recorded at $0.4 \mathrm{~V}$ vs. $\mathrm{Ag} \mid \mathrm{AgCl}$, and sampled at $10 \mathrm{~Hz}$. Here again, the baseline was subtracted.

Electrochemical Impedance Spectroscopy (EIS) traces were obtained to further characterize the device. The range of frequencies was $10 \mathrm{~Hz}-100 \mathrm{kHz}$. The applied potential was a 50 $\mathrm{mV}$ amplitude wave, centered over $0.2 \mathrm{~V}$ vs. Ag|AgCl, the midpeak potential, as indicated by the $\mathrm{CV}$.

Even though the procedures were largely reproducible, minute differences in the experimental conditions were unavoidable: condition of the electrode surface, position of the electrode, trapping of bubbles in the channels, etc. To minimize the effect of these variations on the observed electrochemical behavior, and ensure the physical relevance of our data, the datasets presented in each different section were obtained, unless stated otherwise, from a single experimental session on the same chip, to allow upfront comparison between the different traces. More precisely, the CV traces were obtained just after the AMP measurements had been performed, without any disruption of the fluid flow. The AMP traces were performed before the CV, as this method allowed for the confirmation of an established steady-state and stabilization of the fluidic system, giving rise to the expected shape of the traces (sine, or step, or sawtooth). Similarly, the electrochemical behavior of the system was investigated before each session, by running a control CV in 1 $\mathrm{mM}$ FcMeOH, to check the quality of the device.

\section{Data processing and statistics}

Where applicable, the results obtained from different datasets were averaged, and the variability of the measurement assessed by calculating the standard deviation (SD). The number of individual datapoints for an identical experimental condition is indicated by the value $N$. The significance of the differences observed between two datasets was tested with a double-tailed Student's t-test (assuming the data were normally distributed and that the variances were equal). The criterion for significance was $\mathrm{p}<0.001$, indicated in the text by the symbol .

The deconvolution treatments were performed in Matlab R2012b. All the other processing routines were run in IgorPro 6.34 .

\section{Acknowledgements}

Funding of this work was provided by the EPFL, the EU Ideas program (ERC-2012-AdG-320404). The authors thank the staff of the Center of Micro- and Nanotechnology of EPFL for assistance in the micro-fabrication processes.

\section{Notes and references}

Laboratory of Microsystems, Ecole Polytechnique $\begin{gathered}\text { Fédérale de } \\ \text { Lausanne, } \\ \text { CH-1015 }\end{gathered}$ Lausanne,
$\begin{aligned} & \text { raphael.trouillon@m4x.org } \\ & \text { Electronic Supplementary Information (ESI) available: Additional }\end{aligned}$
details (processing of the CV data, calculation of the electrode 
capacitance from CV background traces, derivation of the relaxation time of the diffusion layer through an analogy with electronics, direct measurement of the MTF with sine concentration waves). See DOI: $10.1039 / \mathrm{b} 000000 \mathrm{x} /$

1. C. T. Culbertson, T. G. Mickleburgh, S. A. Stewart-James, K. A. Sellens, and M. Pressnall, Anal. Chem., 2014, 86, 95-118.

2. Y. Lin, R. Trouillon, G. Safina, and A. G. Ewing, Anal. Chem., 2011, 83, 4369-4392.

3. R. Trouillon, M. K. Passarelli, J. Wang, M. E. Kurczy, and A. G. Ewing, Anal. Chem., 2013, 85, 522-542.

4. Y. Xia and G. M. Whitesides, Annu. Rev. Mater. Sci., 1998, 28, 153184.

5. G. M. Whitesides, E. Ostuni, S. Takayama, X. Jiang, and D. E. Ingber, Annu. Rev. Biomed. Eng., 2001, 3, 335-373.

6. J. Wang, Talanta, 2002, 56, 223-231.

7. J. Rossier, F. Reymond, and P. E. Michel, Electrophoresis, 2002, 23, 858-867.

8. W. R. Vandaveer, S. A. Pasas-Farmer, D. J. Fischer, C. N. Frankenfeld, and S. M. Lunte, Electrophoresis, 2004, 25, 3528-3549.

9. M. Perry, Q. Li, and R. T. Kennedy, Anal. Chim. Acta, 2009, 653, $1-$ 22.

10. R. N. Adams and C. A. Marsden, in Handbook of Psychopharmacology, eds. L. L. Iversen, S. D. Iversen, and S. H. Snyder, Springer US, 1982, pp. 1-74.

11. S. A. Wring and J. P. Hart, Analyst, 1992, 117, 1215.

12. K. L. Adams, M. Puchades, and A. G. Ewing, Annu. Rev. Anal. Chem., 2008, 1, 329-355.

13. V. Sivagnanam and M. A. M. Gijs, Chem. Rev., 2013, 113, 32143247.

14. D. Huh, G. A. Hamilton, and D. E. Ingber, Trends Cell Biol., 2011, 21, 745-754.

15. A. M. Ghaemmaghami, M. J. Hancock, H. Harrington, H. Kaji, and A. Khademhosseini, Drug Discov. Today, 2012, 17, 173-181.

16. D. Huh, Y. Torisawa, G. A. Hamilton, H. J. Kim, and D. E. Ingber, Lab. Chip, 2012, 12, 2156.

17. M. Thompson and R. G. Compton, Anal. Chem., 2007, 79, 626-631.

18. R. G. Compton, A. C. Fisher, R. G. Wellington, P. J. Dobson, and P. A. Leigh, J. Phys. Chem., 1993, 97, 10410-10415.

19. C. Amatore, N. Da Mota, C. Sella, and L. Thouin, Anal. Chem., 2007, 79, 8502-8510.

20. C. Amatore, N. Da Mota, C. Lemmer, C. Pebay, C. Sella, and L. Thouin, Anal. Chem., 2008, 80, 9483-9490.

21. B. D. Bath, D. J. Michael, B. J. Trafton, J. D. Joseph, P. L. Runnels, and R. M. Wightman, Anal. Chem., 2000, 72, 5994-6002.

22. B. J. Venton, K. P. Troyer, and R. M. Wightman, Anal. Chem., 2002, 74, 539-546.

23. J. H. Park, S. N. Thorgaard, B. Zhang, and A. J. Bard, J. Am. Chem. Soc., 2013, 135, 5258-5261.

24. W. Miao, Z. Ding, and A. J. Bard, J. Phys. Chem. B, 2002, 106, 1392-1398.

25. C. Cannes, F. Kanoufi, and A. J. Bard, J. Electroanal. Chem., 2003, 547, 83-91.

26. A. J. Bard and R. L. Faulkner, Electrochemical Methods: Fundamentals and Applications, John Wiley and Sons, Hoboken, NJ, USA, 2nd edn., 2001.
27. K. Aoki, K. Honda, K. Tokuda, and H. Matsuda, J. Electroanal. Chem. Interfacial Electrochem., 1985, 182, 267-279.

28. W. Harreither, R. Trouillon, P. Poulin, W. Neri, A. G. Ewing, and G. Safina, Anal. Chem., 2013, 85, 7447-7453.

29. V. G. Levich, Physiochemical hydrodynamics, Prentice-Hall, Englewood Cliffs, N.J, 1962.

30. W. J. Blaedel and L. N. Klatt, Anal. Chem., 1966, 38, 879-883.

31. H. Matsuda, J. Electroanal. Chem., 1967, 15, 325-336.

32. J. M. Elbicki, D. M. Morgan, and S. G. Weber, Anal. Chem., 1984, 56, 978-985.

33. P. M. Kovach, W. L. Caudill, D. G. Peters, and R. M. Wightman, J. Electroanal. Chem. Interfacial Electrochem., 1985, 185, 285-295.

34. S.-M. Park and J.-S. Yoo, Anal. Chem., 2003, 75, 455 A-461 A.

35. H. E. Jacobse-Geels, M. R. Daha, and M. C. Horzinek, J. Immunol., 1980, 125, 1606-1610.

36. J. L. Dawson and D. G. John, J Electroanal Chem, 1980, 110, 37-47.

37. H. Bruus, Theoretical Microfluidics, Oxford University Press, Oxford, UK, 1st edn., 2008.

38. M. R. Doshi, P. M. Daiya, and W. N. Gill, Chem. Eng. Sci., 1978, 33, 795-804.

39. D. Dutta and D. T. Leighton, Anal. Chem., 2001, 73, 504-513.

40. L. Prandtl, in Verhandlungen des dritten internationalen Mathematiker-Kongresses, 1904, p. 484.

41. J. V. Macpherson and P. R. Unwin, Anal. Chem., 1999, 71, 2939 2944.

42. J. V. Macpherson and P. R. Unwin, Anal. Chem., 1999, 71, 46424648.

43. H. Knutsson and C.-F. Westin, in , 1993 IEEE Computer Society Conference on Computer Vision and Pattern Recognition, 1993. Proceedings CVPR '93, 1993, pp. 515-523.

44. X. Zhang, T. Kashti, D. Kella, T. Frank, D. Shaked, R. Ulichney, M. Fischer, and J. P. Allebach, 2012, vol. 8293, pp. 829307-829307-11.

45. B. Zhang, J. Zerubia, and J.-C. Olivo-Marin, Appl. Opt., 2007, 46, 1819-1829.

46. H. Fujita, D.-Y. Tsai, T. Itoh, K. Doi, J. Morishita, K. Ueda, and A. Ohtsuka, IEEE Trans. Med. Imaging, 1992, 11, 34-39.

47. A. P. Tzannes and J. M. Mooney, Opt. Eng., 1995, 34, 1808-1817.

48. G. D. Boreman, Modulation Transfer Function in Optical and Electro-Optical Systems, SPIE, Bellingham, USA, 2001.

49. J. Yamada and H. Matsuda, J. Electroanal. Chem. Interfacial Electrochem., 1973, 44, 189-198.

50. R. A. Clark, P. B. Hietpas, and A. G. Ewing, Anal. Chem., 1997, 69, 259-263.

51. R. A. Clark and A. G. Ewing, Anal. Chem., 1998, 70, 1119-1125.

52. W. J. Thomas and M. J. Adams, Trans. Faraday Soc., 1965, 61, 668673.

53. A. Einstein, Ann. Phys., 1905, 322, 549-560.

54. B. Zhang, M. L. A. V. Heien, M. F. Santillo, L. Mellander, and A. G. Ewing, Anal. Chem., 2011, 83, 571-577.

55. R. Trouillon and A. G. Ewing, Anal. Chem., 2013, 85, 4822-4828.

56. R. Trouillon, Y. Lin, L. J. Mellander, J. D. Keighron, and A. G. Ewing, Anal. Chem., 2013, 85, 6421-6428.

57. M. E. Rice, G. A. Gerhardt, P. M. Hierl, G. Nagy, and R. N. Adams, Neuroscience, 1985, 15, 891-902. 


\section{For Table of Contents only}

Using a microfluidic/ electrochemical chip, it is shown that voltammetric techniques cannot resolve fast concentration changes as well as amperometry, largely because of the intrinsic relaxation time of the diffusion layer, inducing a dampening of the system response at high frequency.
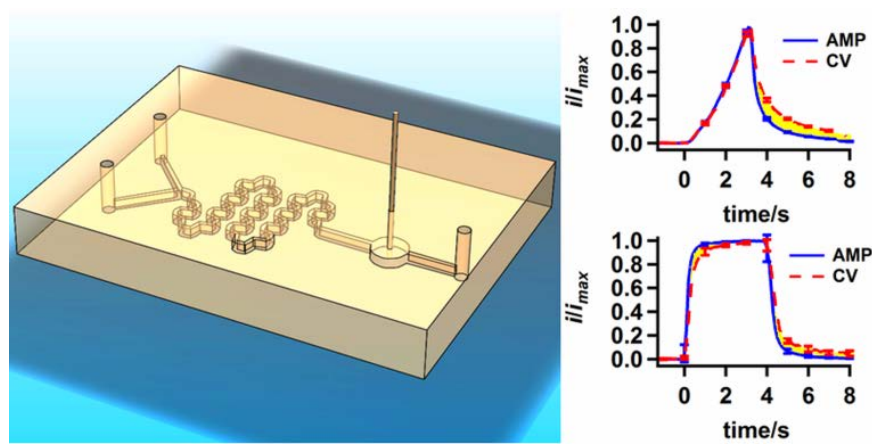